\title{
The accuracy of EUS-FNA in assessing mediastinal lymphadenopathy and staging patients with NSCLC
}

\author{
G. Caddy*, M. Conron\#, G. Wright", P. Desmond*, D. Hart" and R.Y. Chen*
}

ABSTRACT: Optimal management of nonsmall cell lung cancer (NSCLC) depends on tissue diagnosis and accurate staging. Endoscopic ultrasound-guided fine-needle aspiration (EUS-FNA) is minimally invasive and provides cytological confirmation of malignant mediastinal disease. The aim was to assess the accuracy of EUS-FNA in cases of enlarged mediastinal lymphadenopathy (LN) of unknown aetiology and in the staging of NSCLC.

A total of 52 consecutive patients with stage I-IIlb NSCLC or enlarged mediastinal LN of unknown aetiology underwent EUS-FNA. Negative results were confirmed with a surgical procedure: mediastinoscopy, video-assisted thoracic surgery (VATS) or lobectomy with systematic mediastinal lymph node dissection.

In total, 34 patients had EUS-FNA performed for diagnosis, whilst the remaining 18 had EUSFNA for staging. The overall sensitivity, specificity, positive predictive value (PPV), negative predictive value (NPV) and accuracy (95\% confidence interval) were 93\% (77-99), 100\% (78-100), $100 \%$ (87-100), 88\% (63-99) and 95\% (84-99), respectively. When EUS-FNA was used in patients with NSCLC, the sensitivity, specificity, PPV, NPV and accuracy were $92 \%$ (73-99), 100\% (69-100), $100 \%(85-100), 83 \%(51-98)$ and $94 \%(80-99)$, respectively. For mediastinal LN of unknown aetiology, no malignant disease was missed.

Endoscopic ultrasound-guided fine-needle aspiration is an accurate tool for assessing mediastinal lymph node involvement in nonsmall cell lung cancer and in the diagnosis of unexplained mediastinal lymphadenopathy. Endoscopic ultrasound-guided fine-needle aspiration is a minimally invasive procedure that can be used as an adjunct or alternative to mediastinoscopy.

KEYWORDS: Endoscopic ultrasound, endoscopic ultrasound-guided fine-needle aspiration, lung cancer, lymphadenopathy, staging

ung cancer is a major public health problem and the leading cause of cancerrelated death in Australia [1]. Of the $\sim 2,000$ patients presenting with lung cancer each year in Victoria, 85\% have nonsmall cell lung cancer (NSCLC) and the 5-yr survival is only $15 \%$. The poor survival of NSCLC is mainly due to most patients presenting with regionally advanced or metastatic disease not suitable for resection [2], but deficiencies in the delivery of optimal treatment in Australia and abroad may also contribute to the poor outcome [3,4]. In an effort to improve lung cancer care in Australia, guidelines have been drafted to assist in the delivery of clinical services [5]. These guidelines recognise that cytological or histological confirmation of malignancy and accurate tumour, node, metastasis (TNM) staging

For editorial comments see page 400 . is critical for the delivery of effective lung cancer management.

It is reported that $\sim 70 \%$ of lung cancers can be histologically typed bronchoscopically [6], with the remaining tumours requiring an alternative diagnostic procedure. With malignant involvement of the mediastinal lymph nodes being the most common site for metastasis in the $75 \%$ of patients with NSCLC who present with regionally advanced disease [7], this is a common site from which to obtain tissue when bronchoscopic methods fail to establish a tissue diagnosis. If those patients requiring mediastinal sampling for unexplained enlarged lymphadenopathy (LN) or lung masses are also considered, the potential clinical applications for endoscopic ultrasound-guided fine-needle aspiration (EUSFNA) can be appreciated. Furthermore, the ability of EUS-FNA to sample posterior lymph

\section{AFFILIATIONS}

Depts of *Gastroenterology, and \#Respiratory Medicine, and - Cardiothoracic Care Centre, St Vincent's Hospital, Melbourne, Victoria, Australia.

CORRESPONDENCE

G. Caddy

Dept of Gastroenterology

St Vincent's Hospital Melbourne

35 Victoria Parade

Fitzroy

Victoria 3065

Australia

Fax: 61392883590

E-mail: caddygr@svhm.org.au

Received:

August 042004

Accepted after revision:

November 122004
European Respiratory Journal Print ISSN 0903-1936 Online ISSN 1399-3003 
node stations makes the procedure complimentary to mediastinoscopy that best targets the anterior and higher paratracheal stations.

Perhaps the most important application of EUS-FNA is as a minimally invasive technique to stage the mediastinum. In the $50 \%$ of patients with NSCLC who do not have distant metastatic disease, accurate TNM staging of the mediastinum is critical for directing appropriate management. Unfortunately, even with recent technological advances, standard contrast-enhanced computed tomography (CT) is of limited value in assessing the mediastinum in NSCLC, with a recent meta-analysis showing it to be only $57 \%$ sensitive and $82 \%$ specific in correctly identifying malignant LN based on size criteria [2]. The current authors' experience of comparing the accuracy of CT to final mediastinal nodal staging at surgery found a similar specificity to other studies, but with a low sensitivity $(7 \%)$ and positive predictive value (PPV; $8 \%$ ) in pathological N2/N3 disease [8]. While positron emission tomography (PET) scanning has been proven to be a significant advance in the ability to accurately assess the mediastinal lymph nodes (sensitivity 84\%, specificity 89\%) [2], local experience with false-positive and negative studies has led to many clinicians insisting on histological confirmation of malignant involvement [8].

The purpose of this study was to prospectively assess the accuracy of EUS-FNA in the diagnosis of patients with mediastinal LN of unknown aetiology and staging of NSCLC, in a multidisciplinary tertiary referral centre. Data from outside of Australia suggests that the technique is useful, but, to date, there is minimal local data [9].

\section{METHODS}

\section{Study subjects}

Between November 2002 and June 2004, 52 consecutive patients underwent mediastinal sampling by EUS-FNA (fig. 1). Patients were referred from a single multidisciplinary lung clinic (St Vincent's Hospital, Melbourne, Australia) according to an established clinical protocol (fig. 2), with either a mediastinal mass/enlarged LN of unknown aetiology, or suspected stage I-IIIb NSCLC based on clinical suspicion and CT imaging. Enlarged LN was defined as LN $>1 \mathrm{~cm}$ in size detected on CT. Ethics approval was obtained from the St Vincent's Hospital human research ethics committee.

\section{Study design}

All EUS procedures were performed, under conscious sedation, by a single operator using a radial (Olympus fibreoptic GF-UM20 or video GF-UM160; Olympus Australia Pty Ltd, Mt Waverley, Victoria, Australia) and linear echoendoscope (Olympus GF-UC140P; Olympus Australia Pty Ltd). In several patients, the clinical diagnoses, based particularly on CT imaging, were not clear. These patients were initially examined using a radial echoendoscope and, if the suspicion of enlarged mediastinal LN or lung mass was confirmed, FNA was performed using the linear echoendoscope. All EUS-FNA procedures were performed using a 22-gauge Olympus aspiration needle (Olympus Australia Pty Ltd) with a stylet into the lymph node via the biopsy channel under real-time EUS control (fig. 3). A cytopathologist was on site at the time of the procedure to determine if enough cellular material was

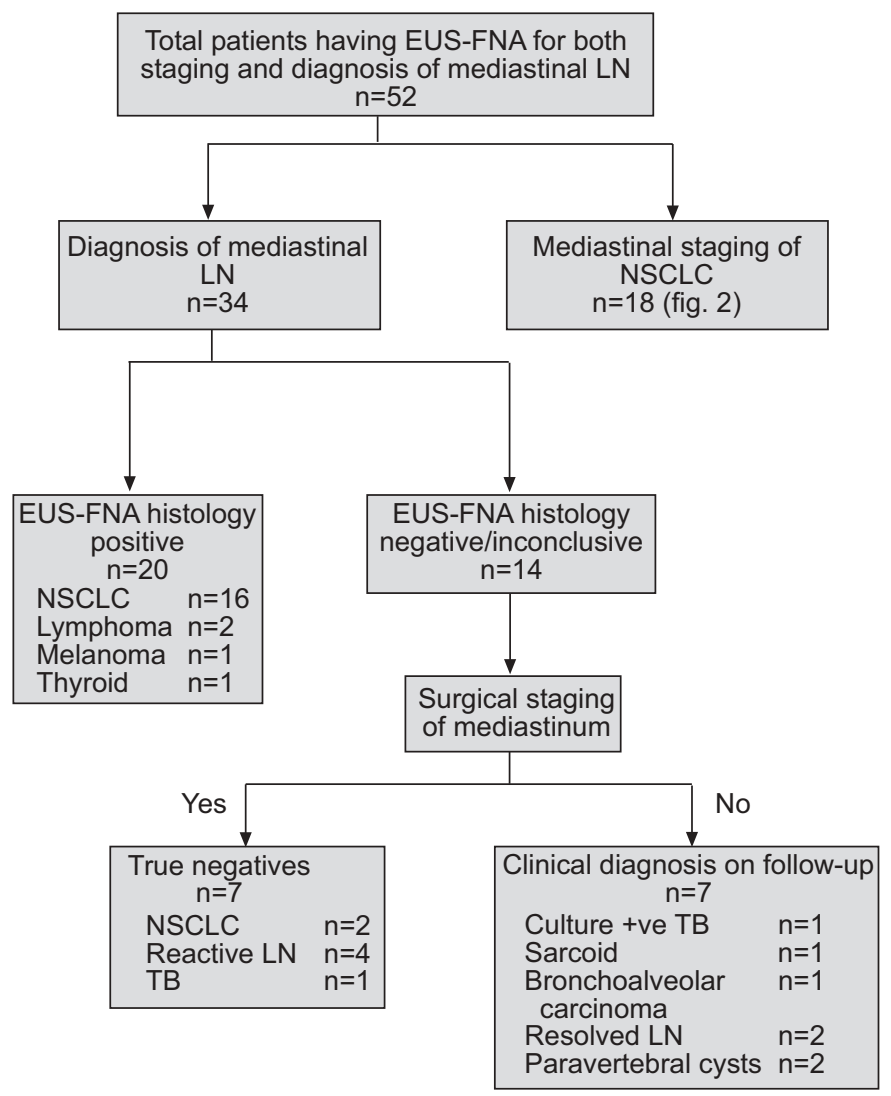

FIGURE 1. Flow diagram showing the total number of patients who underwent endoscopic ultrasound-guided fine-needle aspiration (EUS-FNA) for staging of nonsmall cell lung cancer (NSCLC) and diagnosis of enlarged mediastinal lymphadenopathy (LN) of unknown aetiology. +ve: positive; TB: tuberculosis.

obtained during each FNA and to avoid unnecessary passes of the aspiration needle in cases of adequate samples.

Cytology results were available within $48 \mathrm{~h}$. Final diagnosis was obtained by a questionnaire sent to the referring doctor within 2 months of the EUS procedure. The questionnaire asked the referring doctor for the post-EUS diagnosis, treatment after EUS and accuracy of EUS-FNA compared with final operative diagnosis or staging. Patients who were considered to be surgical candidates with negative cytological specimens on FNA proceeded to either mediastinoscopy or video-assisted thoracic surgery (VATS) \pm lobectomy or pneumonectomy. The choice of surgical procedure was based on surgeon preference. Surgical verification of nondiagnostic EUS-FNA cytology specimens were performed by systematic lymph node dissection.

\section{Analysis}

Based on this information, the sensitivity, specificity, PPV, negative predictive value (NPV) and accuracy of EUS-FNA (including 95\% confidence intervals) were obtained by comparing the FNA histology with the final outcome or staging on the surgical resection specimen. Based on earlier studies demonstrating that a false-positive EUS-FNA cytological result was rare $[10,11]$, positive cytology obtained by EUSFNA was taken as a true positive. 


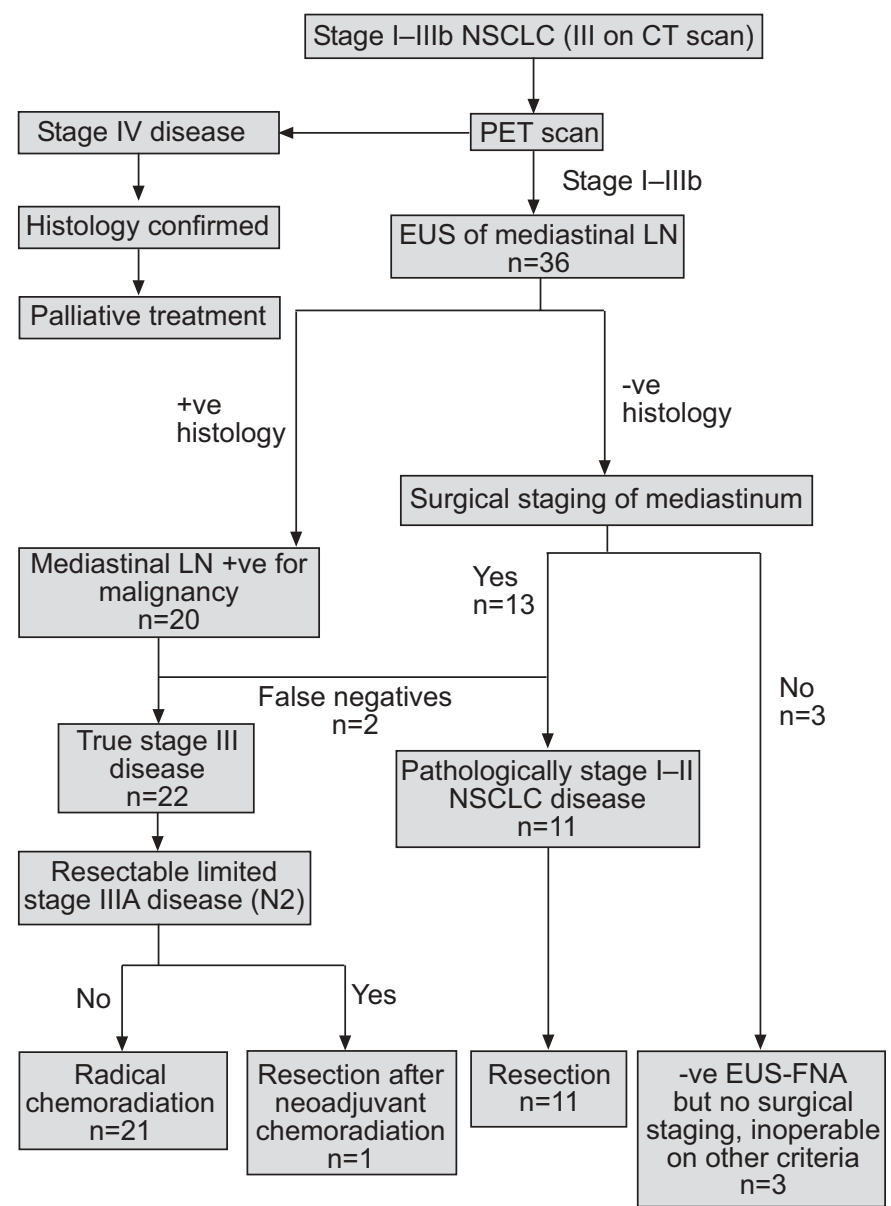

FIGURE 2. Nonsmall cell lung cancer (NSCLC) staging protocol. CT: computed tomography; PET: positron emission tomography; EUS: endoscopic ultrasound; LN: lymphadenopathy; +ve: positive; -ve: negative; FNA: fine-needle aspiration.

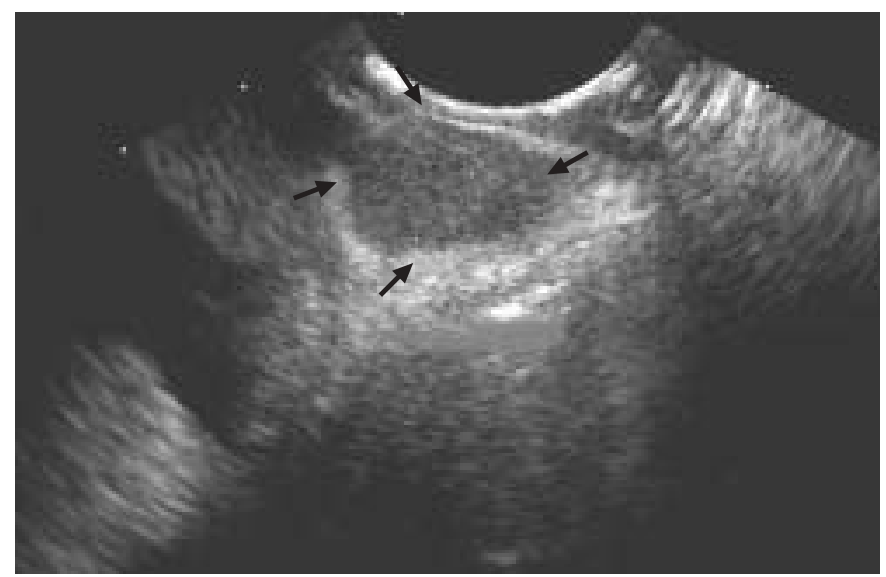

FIGURE 3. Image of a subcarinal lymph node (delineated by arrows) identified on endoscopic ultrasound in a patient with nonsmall cell lung cancer.

\section{RESULTS}

Overall, 72 FNAs were performed on 52 patients (66 lymph nodes and six lung masses). Of these 52 patients, 34 patients (22 males; median age $66 \mathrm{yrs}$ ) had enlarged mediastinal LN and/or a lung mass of unknown aetiology. The remaining 18 patients (15 males; median age $66 \mathrm{yrs}$ ) had an established diagnosis of stage I-IIIb NSCLC.

The mean (range) size of lymph node examined was $22.8 \mathrm{~mm}$ (3-54). The anatomical locations of LN sampled were as follows: 37 subcarinal LNs (station 7), 24 aortopulmonary LNs (station 5), three para-oesophageal LNs (station 8), two paravertebral cysts and six lung masses. There was a mean (range) number of 3.3 passes (1-7) of the aspiration needle per enlarged LN examined.

A total of 18 out of the 34 patients with enlarged mediastinal LN of unknown aetiology were found to have NSCLC, and, in these patients, the EUS-FNA was both a diagnostic and staging procedure. Of those having EUS-FNA performed as a staging procedure for NSCLC $(n=18)$, seven patients had sampling of both subcarinal (station 7) and aortopulmonary (station 5) nodes, one patient had sampling of para-oesophageal nodes (station 8), one patient had sampling of para-oesophageal and subcarinal nodes and, in the remaining eight patients, only sampling of the subcarinal nodes was possible.

Two patients who had a negative EUS-FNA for NSCLC staging were found to be inoperable on other criteria and did not have surgical assessment of the mediastinum to confirm the result. These two patients were excluded from analysis. In 14 of the remaining 16 patients, EUS-FNA correctly staged the mediastinum. In one patient, EUS-FNA incorrectly staged the mediastinum with microscopic disease detected in the station 7 (subcarinal) lymph node following lobectomy. A second patient had incorrect mediastinal staging with both EUS-FNA and mediastinoscopy when, at the time of surgery, direct extension of the tumour into the subcarinal nodes was detected.

Of the 34 patients with enlarged mediastinal LN, both station 7 and 5 nodes were sampled in eight. Station 5 nodes were sampled in six patients and only station 7 nodes were sampled in the remaining 12 patients. In six patients, the FNA was of a mediastinal mass (station 5 nodes were also sampled in one of these patients). In two patients, the FNA was in a paravertebral cyst. In 20 patients (59\%), the EUS-FNAs provided a definitive diagnosis (fig. 1). Of the remaining 14 patients who had a nondiagnostic EUS-FNA, this was found to be a true negative in seven. No surgical staging was performed in the remaining seven patients, but were followed for up to 18 months (mean (range) follow-up period 15 months (8-18)). During the subsequent follow-up period, one patient was diagnosed with tuberculosis (TB) from positive sputum cultures, one patient with sarcoidosis, two patients with resolving paravertebral cysts, two patients with reactive LN (which resolved during follow-up) and one patient with bronchoalveolar carcinoma. In the latter patient, no lymph nodes were sampled and, therefore, the current authors were unable to verify negative mediastinal disease. These seven patients could not be included in analysis, since there was no surgical staging of the mediastinum.

Four of the 14 nondiagnostic FNA specimens contained insufficient cellular material for diagnostic purposes, whereas the remaining 10 specimens contained sufficient cellular material to enable the cytopathologist to ascertain that there 
were no malignant cells within the specimen. These four insufficient FNA samples occurred in the following: one FNA of a paravertebral cyst; one FNA of a lung mass followed by VATS, which confirmed benign disease; one FNA with final diagnosis of bronchoalveolar carcinoma; and one FNA of subcarinal LN and mediastinoscopy confirmed benign LN.

Overall EUS-FNA gives a sensitivity, specificity, PPV, NPV and accuracy (95\% confidence interval) of 93\% (77-99), 100\% (78-100), 100\% (87-100), 88\% (63-99) and 95\% (84-99), respectively. Looking at specific indications, when EUS-FNA was used in patients with known or suspected NSCLC (this includes patients with enlarged mediastinal LN with an eventual diagnosis of NSCLC $(n=34))$, the sensitivity, specificity, PPV, NPV and accuracy were 92\% (73-99), 100\% (69-100), $100 \%(85-100), 83 \%(51-98)$ and 94\% (80-99), respectively. Importantly, for patients presenting with enlarged mediastinal LN of unknown aetiology, no malignant disease was missed by EUS-FNA. If the results are analysed on an intention-to-treat assumption, then the characteristics of EUS-FNA for investigating enlarged mediastinal LN of unknown aetiology are: sensitivity $87 \%$ (66-97), specificity $100 \%$ (72-100), PPV $100 \%$ (83-100), NPV 79\% (49-95) and accuracy of 91\% (76-98). The overall test characteristics of EUS-FNA (including patients with enlarged mediastinal LN and NSCLC) on an intention-totreat basis are: sensitivity $79 \%(61-91)$, specificity $100 \%(82-$ $100)$, PPV $100 \%$ (87-100), NPV 73\% (52-88) and accuracy of $87 \%$ (74-94). These results are on the assumption that EUSFNA correctly identified benign mediastinal disease in patients with an eventual diagnosis of resolved LN and paravertebral cysts, and that EUS-FNA incorrectly identified the mediastinum of patients with an eventual diagnosis of $\mathrm{TB}$, sarcoidosis and NSCLC (including patients that did not go on to have surgical staging of the mediastinum due to other comorbid illness).

\section{DISCUSSION}

In the current study, the experience of EUS-FNA in the assessment of enlarged mediastinal LN and staging of stage I$\mathrm{IIIb}$ NSCLC was reported. There have been several published prospective studies on the use of EUS-FNA in patients with enlarged mediastinal LN. The present study differs from those already in the literature, in that there was a greater number of patients overall with NSCLC $(n=36)$ and fewer patients with benign disease. Also, in contrast to other studies, there were relatively few patients (three out of 52) with a prior history of cancer. In the study by FRITSCHER-RAVENS et al. [12], approximately one third of patients (52 out of 153) examined by EUS had a previous diagnosis of cancer. The same study found EUS-FNA to be more accurate when used in patients with a previous diagnosis of cancer compared to patients without (sensitivity $97 \%$ with cancer versus $88 \%$ without cancer). In the current authors' centre, the main referral base is from chest physicians referring patients with a suspicion of lung cancer (either for staging or to obtain a tissue diagnosis). Although EUS was also performed on patients with benign disease, this group made up the minority of the total number of procedures performed by the current authors. In the study by WIERSEMA et al. [13], which evaluated EUS-FNA in 82 patients with enlarged mediastinal LN, more patients had an eventual diagnosis of benign disease ( 25 out of 82 ) compared with the current patients (12 out of 49). Therefore, in the current study, data are provided that support the accuracy of EUS-FNA, predominately in patients with no personal history of cancer, with known or suspicion of a primary diagnosis of NSCLC. The current authors found EUS to be a safe, minimally invasive procedure that can provide accurate information about both benign and malignant mediastinal disease. Based on the present results, EUS-FNA is a useful addition to existing radiological investigations and surgical techniques that have been previously validated for the assessment of mediastinal disease.

The ability to access nodes from the posterior mediastinal stations makes EUS-FNA a complimentary procedure to mediastinoscopy. Anterior mediastinoscopy is useful in sampling nodes in the superior mediastinum. In particular, station 2 right (high paratracheal), station 4 right (lower paratracheal), station 5 (aortopulmonary) and station 6 (paraaortic) can be accessed. Cervical mediastinoscopy is performed more often and can sample station 2 left and right, station 4 left and right, and station 7 (subcarinal). An extended cervical mediastinoscopy can reach stations 5 and 6 . VATS can access most lymph node stations, but usually only on one side. EUS is particularly suited to examining the left-sided mediastinal lymph nodes, especially stations 4, 5, 8 and 9, and the subcarinal area (station 7). However, EUS cannot reliably image anterior stations (stations 3 and 6) and more distant lymph node sites, such as lobar (station 12) and interlobar sites (station 11) [14]. Cervical mediastinoscopy is the most wellvalidated method of obtaining histological evidence of mediastinal involvement in NSCLC, having been shown to be $87 \%$ sensitive and $100 \%$ specific when performed for this indication. However, its high cost, invasive nature and recognised complication rate of $0.5-2 \%$ means that the procedure is often avoided [15, 16]. EUS-FNA is a safe, less invasive alternative to mediastinoscopy, which is also complimentary, offering access to nodal stations that a single surgical approach would find more difficult to access [11, 17-19]. While EUS-FNA has not been directly compared with transbronchial FNA, the latter has recognised limitations, being only $50-70 \%$ sensitive and $92-100 \%$ specific in assessing malignant mediastinal lymph node involvement in NSCLC and carrying a complication rate of $2-5 \%[20,21]$. The suitability of EUS-FNA for staging of NSCLC is further highlighted by the lack of PET centres in Australia and overseas, requiring many clinicians to perform more invasive mediastinoscopy to histologically prove stage III disease.

Approximately $40 \%$ of patients with NSCLC present with metastatic disease outside the chest (stage IV) and are not suitable for radical treatment [7]. A similar proportion will have locally advanced disease, and, in selected patients with good performance status, the combination of surgery with neoadjuvant chemotherapy (stage IIIa) [22-25] and chemotherapy with radiation (stage IIIb) [26, 27] may provide a small survival advantage. The remaining $20 \%$ will have early (stage I or II) disease and may be suitable for surgery, if competing smoking-related comorbidities do not preclude a radical procedure. Unfortunately, half of those who have what is considered to be a curative resection relapse at a distant site and die of their disease, with the remainder of this surgically treated group forming the vast majority of survivors at 5 yrs 
[28]. With malignant involvement of the mediastinum being the factor that most commonly determines resectability, it is clear why correct staging of this site is critical and why guidelines recommend that exclusion from a potentially curative resection be based on histological evidence of mediastinal involvement. Of equal importance is the avoidance of futile thoracotomies in this high-risk surgical population by detecting radiologically unsuspected stage III mediastinal disease.

The limitations of relying on CT to detect mediastinal LN, based on size criteria, have been previously well described [2, 14]. PET scanning is a significantly better tool to accurately assess mediastinal lymph nodes (sensitivity $84 \%$, specificity $89 \%$ ), but is also limited by a significant number of false negative and positive results [29, 30]. A recently published study suggests that co-registered CT/PET has a significantly better sensitivity and specificity than PET alone in assessing mediastinal lymph node involvement in NSCLC [31], but, currently, few PET centres have the capability to perform these studies.

Despite these considerable advances in the radiological assessment of the mediastinum in NSCLC, variable local experience with false positive and negative studies has led to the development of lung cancer management guidelines emphasising the importance of histological confirmation of staging in NSCLC. In the current study, the overall sensitivity, specificity, PPV, NPV and accuracy of $93 \%, 100 \%, 100 \%, 88 \%$ and $95 \%$, respectively, compares favourably with a combined meta-analysis of 215 patients who underwent EUS-FNA of mediastinal LN and masses for the staging of NSCLC, which reports a sensitivity, specificity, PPV and NPV of $88 \%, 91 \%$, $98 \%$ and $77 \%$, respectively [32]. HERNANDEZ et al. [33] demonstrated an overall accuracy of $84 \%$ using EUS-FNA in a series of 59 patients (34\% for staging of NSCLC) with mediastinal LN. In the current study, there were two definite false negatives, where N2 disease was detected in the surgically resected specimen (one microscopic disease). One of these patients also had negative mediastinoscopy. Nine patients had a negative EUS-FNA, but no surgical staging of their mediastinum and, therefore, were excluded from analysis. Of these nine patients, three patients had NSCLC and were found to be inoperable, based on further staging procedures (two VATS and one PET), and, therefore, the mediastinum was not sampled to verify the EUS-FNA result. One of these patients was diagnosed with bronchoalveolar carcinoma, and histology was confirmed on a core biopsy at VATS. No mediastinal nodes were sampled for surgical comparison. Metastatic spread in this condition is usually via alveoli rather than lymphatic spread, and mediastinal involvement is not common. Of the remaining six patients who had a negative EUS-FNA and no surgical staging of LN, one patient was diagnosed with TB on a positive sputum culture and one patient with sarcoidosis. Two patients were diagnosed with paravertebral cysts, both of which resolved on subsequent follow-up. Two patients had reactive LN. These six patients had a clinical illness consistent with the established diagnoses, and none has gone on to develop malignant disease in $>18$ months of follow-up. The current study, like previous reports from other centres, found EUS-FNA to be accurate in the diagnosis of mediastinal LN and staging of NSCLC. Although false negatives are reported for both the diagnostic and staging arm of the present study, the inaccuracies did not necessary adversely affect patient management. The presence of micrometastatic disease detected in surgically resected mediastinal lymph nodes is a recognised limitation of mediastinoscopy and, based on current data [34], does not indicate inappropriate treatment. It is recognised that patients with micrometastatic N2 mediastinal disease receiving initial surgical treatment have a better prognosis than those who do not have a resection and receive a significant benefit from postoperative chemotherapy [34]. The false negative for tuberculous mediastinal LN is not unexpected, given the small volume of tissue obtained, and, while EUS-FNA would not be recommended if this diagnosis was suspected, it did allow exclusion of malignant pathology that often coexists with tuberculous reactivation. When EUS-FNA was used in patients with enlarged mediastinal LN, no malignant disease was missed.

In summary, the current study demonstrates that endoscopic ultrasound-guided fine-needle aspiration is a useful tool for the assessment of enlarged mediastinal lymphadenopathy of unknown aetiology and staging of nonsmall cell lung cancer. The current authors found endoscopic ultrasound to be a safe procedure that provided accurate information about both benign and malignant mediastinal disease. Endoscopic ultrasound-guided fine-needle aspiration is a complimentary procedure to mediastinoscopy and is particularly useful for centres managing significant numbers of patients with lung cancer without access to positron emission tomography.

\section{REFERENCES}

1 Giles G, Thursfield V. Canstat: a digest of facts and figures on cancer. Cancer in Victoria, 2002. www.accv.org.au/ cancer1/facts/pdfs/canstats/canstat_40_cancer_vic_2002.pdf. Date last updated: February 1 2004. Date last accessed: December 222004.

2 Toloza E, Harpole L, McCrory D. Noninvasive staging of non-small cell lung cancer: a review of the current evidence. Chest 2003; 123: Suppl. 1, 137S-146S.

3 Brown JS, Eraut D, Trask C, Davison AG. Age and the treatment of lung cancer. Thorax 1996; 51: 564-568.

4 Fergusson RJ, Gregor A, Dodds R, Kerr G. Management of lung cancer in South East Scotland. Thorax 1996; 51: 569-574.

5 National Health and Medical Research Council. Clinical practice guidelines for the prevention, diagnosis and management of lung cancer (cp97). www.nhmrc.gov.au/ publications/synopses/cp97syn.htm. Date last updated: July 15 2004. Date last accessed: December 222004.

6 Schreiber G, McCrory DC. Performance characteristics of different modalities for diagnosis of suspected lung cancer: summary of published evidence. Chest 2003; 123: Suppl. 1, 115S-128S.

7 Ries LA, Eisner MP, Kosary CL, et al. SEER Cancer Statistics Review, 1975-2001. Bethesda, National Cancer Institute, 2001.

8 Daniels M. Follow-up after completely resected non-small cell lung cancer: can PET predict prognosis? Advanced Medical Science, University of Melbourne, 2004. 
9 Fickling W, Wallace M. EUS in lung cancer. Gastrointest Endosc 2002; 56: Suppl. 4, S18-S21.

10 Gress FG, Savides TJ, Sandler A, et al. Endoscopic ultrasonography, fine-needle aspiration biopsy guided by endoscopic ultrasonography, and computed tomography in the preoperative staging of non-small-cell lung cancer: a comparison study. Ann Intern Med 1997; 127: 604-612.

11 Fritscher-Ravens A, Soehendra N, Schirrow L, et al. Role of transoesophageal endosonography-guided fine-needle aspiration in the diagnosis of lung cancer. Chest 2000; 117: 339-345.

12 Fritscher-Ravens A, Sriram PV, Bobrowski C, et al. Mediastinal lymphadenopathy in patients with or without previous malignancy: EUS-FNA-based differential cytodiagnosis in 153 patients. Am J Gastroenterol 2000; 95: 2278-2284.

13 Wiersema MJ, Vazquez-Sequeiros E, Wiersema LM. Evaluation of mediastinal lymphadenopathy with endoscopic US-guided fine-needle aspiration biopsy. Radiology 2001; 219: 252-257.

14 Leblanc J, Espada R, Ergun G. Non-small cell lung cancer staging techniques and endoscopic ultrasound: tissue is still the issue. Chest 2003; 123: 1718-1725.

15 Luke WP, Pearson FG, Todd TR, Patterson GA, Cooper JD. Prospective evaluation of mediastinoscopy for assessment of carcinoma of the lung. J Thorac Cardiovasc Surg 1986; 91: $53-56$.

16 Gossot D, Toledo L, Fritsch S, Celerier M. Mediastinoscopy versus thoracoscopy for mediastinal biopsy. Results of a prospective nonrandomized study. Chest 1996; 110: 1328-1331.

17 Mishra G, Sahai AV, Penman ID, et al. Endoscopic ultrasound with fine-needle aspiration in the diagnosis and staging of lung cancer. Endoscopy 1999; 31: 377-382.

18 Silvestri GA, Hoffman BJ, Bhutani MS, et al. Endoscopic ultrasound with fine-needle aspiration in the diagnosis and staging of lung cancer. Ann Thorac Surg 1996; 61: 1441-1445.

19 Wallace MB, Silvestri GA, Sahai AV, et al. Endoscopic ultrasound-guided fine needle aspiration for staging patients with carcinoma of the lung. Ann Thorac Surg 2001; 72: 1861-1867.

20 Kramer H, Groen HJ. Current concepts in the mediastinal lymph node staging of nonsmall cell lung cancer. Ann Surg 2003; 238: 180-188.

21 Bilaceroglu S, Cagiotariotaciota U, Gunel O, Bayol U, Perim K. Comparison of rigid and flexible transbronchial needle aspiration in the staging of bronchogenic carcinoma. Respiration 1998; 65: 441-449.

22 Rosell R, Gomez-Codina J, Camps C, et al. A randomized trial comparing preoperative chemotherapy plus surgery with surgery alone in patients with non-small-cell lung cancer. N Engl J Med 1994; 330: 153-158.

23 Roth JA, Fossella F, Komaki R, et al. A randomized trial comparing perioperative chemotherapy and surgery with surgery alone in resectable stage IIIA non-small-cell lung cancer. J Natl Cancer Inst 1994; 86: 673-680.

24 Roth JA, Atkinson EN, Fossella F. Long-term follow-up of patients enrolled in a randomized trial comparing perioperative chemotherapy and surgery with surgery alone in resectable stage IIIA non-small-cell lung cancer. Lung Cancer 1998; 21: 1-6.

25 Rosell R, Gomez-Codina J, Camps C, et al. Preresectional chemotherapy in stage IIIA non-small-cell lung cancer: a 7year assessment of a randomized controlled trial. Lung Cancer 1999; 26: 7-14.

26 Non-small Cell Lung Cancer Collaborative Group. Chemotherapy in non-small cell lung cancer: a metaanalysis using updated data on individual patients from 52 randomised clinical trials. BMJ 1995; 311: 899-909.

27 Pritchard RS, Anthony SP. Chemotherapy plus radiotherapy compared with radiotherapy alone in the treatment of locally advanced, unresectable, non-small-cell lung cancer. A meta-analysis. Ann Intern Med 1996; 125: 723-729.

28 Colice G, Rubins J, Unger M. American College of Chest Physicians. Follow-up and surveillance of the lung cancer patient following curative-intent therapy. Chest 2003; 123: Suppl. 1, 272S-283S.

29 Vansteenkiste JF. PET scan in the staging of non-small cell lung cancer. Lung Cancer 2003; 42: Suppl. 1, S27-S37.

30 Annema J, Hoekstra O, Smit E, Veselic M, Versteegh MI, Rabe KF. Towards a minimally invasive staging strategy in NSCLC: analysis of PET positive mediastinal lesions by EUS-FNA. Lung Cancer 2004; 44: 53-60.

31 Lardinois D, Weder W, Hany TF, et al. Staging of nonsmall-cell lung cancer with integrated positron-emission tomography and computed tomography. $N$ Engl J Med 2003; 348: 2500-2507.

32 Toloza EM, Harpole L, Detterbeck F, McCrory DC. Invasive staging of non-small cell lung cancer: a review of the current evidence. Chest 2003; 123: Suppl. 1, 157S-166S.

33 Hernandez LV, Mishra G, George S, Bhutani MS. A descriptive analysis of EUS-FNA for mediastinal lymphadenopathy: an emphasis on clinical impact and false negative results. Am J Gastroenterol 2004; 99: 249-254.

34 Arriagada R, Bergman B, Dunant A, et al. International Adjuvant Lung Cancer Trial Collaborative Group. Cisplatin-based adjuvant chemotherapy in patients with completely resected non-small-cell lung cancer. $N$ Engl J Med 2004; 350: 351-360. 